\title{
Representações sociais e práticas corporais de rejuvenescimento para mulheres de meia-idade ${ }^{1}$
}

\author{
Social representations and bodily practices of rejuvenation for middle-aged women
}

\author{
Adriana de Aguiar ${ }^{2}$ \\ Brigido Vizeu Camargo 3 \\ Andrea Barbará da Silva Bousfield ${ }^{4}$
}

\begin{abstract}
RESUMO: Utilizando-se do aporte teórico das representações sociais, este estudo teve como objetivo analisar a relação entre as representações sociais (RS) do rejuvenescimento com práticas corporais relativas a este objeto em mulheres de meia idade, enfatizando atitudes, crenças e normas como elementos de mediação entre os dois construtos. Um levantamento de dados foi realizado com 100 mulheres de meia-idade ( $M=49$ anos e 8 meses; $D P=6$ anos e 9 meses) e o instrumento utilizado consistiu em um questionário estruturado. Foram consideradas 8 práticas corporais de rejuvenescimento, divididas em duas modalidades: práticas não invasivas (práticas alimentares, exercícios físicos, cosméticos/cremes anti-idade e tinturas para cabelo) e práticas minimamente invasivas e invasivas (Botox, peeling químico, preenchimento cutâneo e cirurgia plástica). Foram realizadas análises descritivo-relacionais, com auxílio do software SPSS. Os resultados mostram ampla aceitação de práticas corporais de rejuvenescimento, com maior aprovação de práticas não invasivas do que minimamente invasivas e invasivas. 0 rejuvenescimento enquanto objeto de saúde e bem-estar subjetivo foi uma representação compartilhada por todas as mulheres, no entanto, enquanto objeto de beleza, observaram-se crenças positivas entre as mulheres com atitudes mais favoráveis às práticas corporais de rejuvenescimento e negativas entre as desfavoráveis a estas práticas. As atitudes, crenças e normas referentes às práticas de rejuvenescimento se relacionaram à maior intencionalidade de adoção destas práticas, assim como a adoção concreta. Os resultados sugerem que há relação entre RS do rejuvenescimento e as práticas corporais relativa a este objeto, embora sejam necessários outros estudos para abordar em detalhes esta relação.
\end{abstract}

Palavras-chaves: representações sociais; rejuvenescimento; práticas corporais.

\begin{abstract}
Using the theoretical framework of social representations (SR), this research aimed at analysing the relationship between the SR of rejuvenation and their associated body practices of rejuvenation among middle-aged women, emphasizing attitudes, beliefs and norms as elements of negotiation between the two constructs. A survey was conducted with 100 middle-aged women ( $M=49$ years and 8 months; SD= 6 years and 9 months) and it used a structured questionnaire. It was considered 8 rejuvenation body practices, divided into two modalities: noninvasive practices (feeding practices, physical exercises, cosmetics/ anti-aging creams and dyeing of white hair) and minimally invasive and invasive practices (botox, chemical peels, dermal filling and plastic surgery). Descriptive-relational analyzes were performed with the support of the software SPSS. The results suggest wide acceptation of rejuvenation body practices, in special the non-invasive ones when compared with invasive and minimally invasive practices. The rejuvenation as an object of health and subjective well-being was a representation shared by all
\end{abstract}

\footnotetext{
${ }^{1}$ Agência de fomento: Coordenação de Aperfeiçoamento de Pessoal de Nível Superior, CAPES, Brasil. Conselho Nacional de Desenvolvimento Científico e Tecnológico, CNPq

2 Doutora em Psicologia pela Universidade Federal de Santa Catarina. Psicóloga da Prefeitura Municipal de Florianópolis Florianópolis, SC - Brasil. E-mail: adrianadeaguiar@yahoo.com.br.

${ }^{3}$ Doutor em Psicologia Social. Professor do Programa de Pós-Graduação em Psicologia da Universidade Federal de Santa Catarina - Florianópolis, SC, Brasil.

4 Doutora em Psicologia. Professora do Programa de Pós-Graduação em Psicologia da Universidade Federal de Santa Catarina - Florianópolis, SC, Brasil.
} 
women, however, as an object of beauty, positive beliefs were observed among women with attitudes more favorable to body rejuvenation practices and negative among those unfavorable to these practices. The attitudes, beliefs and norms regarding rejuvenation practices were related to the greater intentionality of adopting these practices, as well as the concrete adoption. The results suggest that there is a relationship between RS of rejuvenation and body practices regarding this object, although further studies are needed to address this relationship in detail.

Keywords: social representations; rejuvenation; body practices.

\section{Introdução}

Como teorias sociais práticas sobre os objetos sociais, as representações sociais referem-se a uma forma de conhecimento vinculada à experiência que o produziu e servem para que os indivíduos ajam sobre o mundo e sobre o outro (Jodelet, 1989). Elas modulam simbolicamente o contexto no qual um comportamento ocorre (Moscovici, 1961, 1978) e também são utilizadas na justificação destes comportamentos, como forma de interpretar a realidade e tornar o mundo compreensível (Vala, 2006). Desta forma, o estudo das práticas sociais permite conhecer as representações sociais que as organizam.

As definições propostas na literatura a respeito de práticas sociais, em geral, as referem como um conjunto complexo de ações ou comportamentos que são legitimados socialmente, ou seja, são conectados ao contexto social que os produziu (Lheureux, Lo Monaco \& Guimelli, 2011; Flament, 2001; Abric, 1994). Sá (1994) as definem como um conjunto de comportamentos e ações que são determinados por representações sociais, diferindo-as da noção de comportamento, ligado mais à concepção behaviorista que maximiza fatores situacionais na determinação da ação em detrimento de elementos simbólicos.

A relação entre o pensamento leigo e as práticas ainda permanece indefinida no campo de estudo das representações sociais (Campos, 2003). Alguns autores enfatizam a relação de influência das representações sobre as práticas (Moscovici, 1961, 1978), outros o efeito das práticas sobre as representações (Morot \& Sales-Wuillemin, 2008; Mamontoff, 2008; Guimelli, 1998) e uma terceira perspectiva, considerada predominante por autores como Almeida, Santos e Trindade (2000), considera a ideia de interação mútua entre os dois constructos. Wagner $(2015,2003)$, por sua vez, questiona a distinção entre representações sociais e comportamento, ao considerar que as ações fazem parte do conteúdo representacional frente aos objetos, portanto não haveria uma separação entre representações sociais e práticas.

O estudo da relação entre representações sociais e práticas implica em considerar a articulação entre dois constructos de naturezas distintas. O primeiro caracteriza-se por sua natureza social e compartilhada, enquanto o segundo refere-se a ações, que são caracterizadas por uma expressão individual. Alguns autores consideram que a articulação entre representações sociais e atitudes permite considerações a respeito da relação entre representações sociais e comportamentos ou práticas (Camargo \& Bousfield, 2014; Wackelke \& Camargo 2007).

Na perspectiva da cognição social, a atitude tem amplo respaldo na literatura como uma variável que permite integrar cognição e comportamento, na medida em que funcionaria como predisposição à ação (Ajzen, 1991, 2001, 2005; Ajzen \& Madden, 1986; Fishbein \& Ajzen, 1975). Essa característica permite considerá-la como um constructo 
pertinente ao estudo da relação entre representações sociais e práticas, apresentando-se como um intermediário importante nesta relação (Moliner \& Tafani, 1997).

As atitudes, de uma forma geral, são definidas como um constructo psicológico de natureza avaliativa (Neiva \& Mauro, 2011; Lima, 2006; Ajzen, 2005), consistindo em disposições para responder favorável ou não favoravelmente a um objeto (Doise, 2001; Stroebe \& Stroebe, 1995). A avaliação implica uma tomada de posição, que por sua vez, está sempre inserida em um uma dinâmica social presente no contexto do sujeito, em que constantemente sua manifestação é demandada nos processos inerentes às relações sociais e pertenças grupais (Doise, 2001).

Moscovici $(1961,1978)$ destaca o caráter afetivo das atitudes, situando-as como um dos componentes que compoem as representações sociais, juntamente com a informação sobre o objeto e o campo. Este autor considera que, em geral, a atitude tende a ser a dimensão mais presente, possivelmente sendo a primeira em uma hierarquia genética, uma vez que somente se busca informações e se elabora uma ideia organizada sobre o objeto após ter tomado uma posição e em função deste posicionamento. Elas são elementos primários na formação das representações sociais, o que permite considerá-las como organizadoras das cognições que compõe o campo representacional associado ao objeto, configurando-se em sistemas de significação que virão a orientar os comportamentos (Parales-Quenza \& Vizicaíno-Gutiérrez, 2007).

Embora as representações sociais sejam formadas a partir da tomada de posição em relação ao objeto, a formação de uma atitude também exige que se tenha alguma informação sobre o mesmo (Moscovici, 1961, 1978), indicando uma relação de reciprocidade entre os dois constructos. As atitudes são alicerçadas em crenças (Ajzen, 2006; Fishbein \& Ajzen, 1975), o que pressupõe uma regulação dos processos cognitivos por fenômenos societários. Moliner e Tafani (1997), por sua vez, afirmam que os componentes avaliativos de uma representação formam a estrutura subjacente de uma atitude, ou seja, as primeiras permitem explicar as bases cognitivas das últimas.

Apesar da importância dos elementos avaliativos na relação entre representações sociais e práticas, o conhecimento do senso comum é composto por fragmentos de uma variedade de elementos, de natureza cognitiva e social (Jodelet, 2001). Assim, é possível considerar que, além das atitudes, o campo representacional em torno das práticas esteja sujeito a outras regulações. Doise $(1985,2014)$, apreendendo o conceito de metassistema de Moscovici $(1961,1978)$, defende a necessidade de estudar a relação entre as regulações sociais e o funcionamento cognitivo, que por sua vez, permite explicar os comportamentos. A regulação social sobre o comportamento pressupõe considerar que as normas influenciam as escolhas dos modos de pensar das pessoas e dos grupos, portanto elas podem ser consideradas importantes elementos constituintes das representações sociais.

A relação entre o contexto normativo e a ação é enfatizada por alguns modelos teóricos provenientes da Cognição Social, tais como a Teoria da Ação Refletida (Ajzen \& Fishbein 1975) e do Comportamento Planejado (Ajzen, 1991, 2001, 2005; Ajzen \& Madden, 1986). Considerando especificamente a relação entre representações sociais e práticas, Camargo e Bousfield (2014) propuseram um modelo teórico que reconhece as normas subjetivas, juntamente com as atitudes, como importantes variáveis de mediação entre os dois construtos.

Autores como Thøgersen (2006) também sustentam que o poder prescritivo das normas pode variar conforme seus graus de internalização. Normas mais internalizadas 
envolvem regulações sociais mais prescritivas, enquanto as menos internalizadas mais descritivas. $\mathrm{O}$ autor classifica as normas mais prescritivas em um contínuo de acordo com o nível de internalização e integração ao self, dividindo-as em normas subjetivas e pessoais. As normas subjetivas são impostas pelas expectativas da adminstração de recompensas e punições externas, ou seja, referem-se a uma pressão social percebida no que se refere à aprovação/reprovação de pessoas significativas em se adotar um comportamento (Ajzen \& Fishbein, 1975). Já as normas pessoais caracterizam-se por sua internalização pelos indivíduos e correspondem às expectativas que eles possuem em relação a si próprios, experienciadas como um sentimento de obrigação moral, a partir dos valores adotados (Bertoldo, 2013). Assim, explicar as ações humanas a partir da perspectiva das representações sociais requer considerar os determinantes sociais e societais que as compõe e que permitem a contextualização de crenças, atitude e comportamentos frente aos objetos.

Neste estudo se teve o objetivo de relacionar representações sociais com práticas sociais a partir do objeto Rejuvenescimento. O rejuvenescimento é considerado uma forma de mascarar a velhice e atenuar os impactos do processo natural de envelhecimento (StuartHamilton, 2002), sendo socialmente representando como um objeto de saúde, de beleza e ligado a condições psicológicas e espirituais (Aguiar, 2016; Castro, Aguiar, Berri \& Camargo, 2016; Berri, Castro \& Camargo, 2016; Torres, 2010). Enquanto objeto de beleza, envolve um variado leque de intervenções, procedimentos e produtos, que são utilizados para a obtenção de uma aparência mais próxima a dos jovens. O emprego de técnicas mais intervencionistas é associado a um maior nível de vaidade (Chasteen, Bashir, Gallucci \& Visekruna, 2011), contudo estudos têm apontado uma predileção das mulheres por práticas menos invasivas a opções cirúrgicas, devido ao efeito menos drástico das primeiras sobre a aparência, mantendo a ideia de um envelhecimento natural (Pereira \& Penalva, 2011; Chasteen et al., 2011; Muise \& Desmarais, 2010; Clarke, Repta \& Griffin, 2007; Clarke \& Griffin, 2007; Teixeira et al., 2007). Além disso, práticas em interface com a saúde, como exercícios físicos e boa alimentação, também são valorizadas como estratégias para obter bem-estar físico e jovialidade (Aguiar, 2016; Castro et al, 2016; Berri et al, 2016; Castro, Antunes, Brito \& Camargo, 2016; Pereira \& Penalva, 2011).

A juventude corresponde a um valor na cultura brasileira, o que torna o "ser jovem" um sinônimo de beleza, atividade e vigor, tornando-se, nos dias atuais, uma preocupação comum entre pessoas de diferentes faixas etárias (Pereira \& Penalva, 2011; Muise \& Desmarais, 2010; Teixeira et al., 2007). Embora as mulheres não sejam as únicas a se preocuparem com as transformações corporais provocadas pelo envelhecimento, elas podem sofrer maior impacto em relação à visibilidade dos seus sinais e serem mais suscetíveis a intervenções sobre o corpo, já que apresentam maior insatisfação com a imagem corporal (Muth \& Cash, 1997) e são mais sensíveis às influências sociais em relação à aparência quando comparadas aos homens (Camargo, Justo \& Jodelet, 2010). Elas buscam intervenções de beleza e rejuvenescimento visando o combate à invisibilidade social, o aumento da autoestima, a manutenção da competitividade a nível sexual e profissional (Aguiar, 2016; Berri et al, 2016; Clarke \& Griffin, 2008), bem como uma estratégia para amenizar a ansiedade em relação ao envelhecimento (Slevec \& Tiggemann, 2010), este associado à falta de atratividade (Ehlinger-Martin, Cohen-Letessier, Taïeb, Azoulay \& du Crest, 2015; Yaworsky et al, 2014). 
Embora as práticas de rejuvenescimento venham recebendo cada vez mais destaque no cotidiano e exista um contingente expressivo de pesquisas em psicologia social abordando as concepções leigas sobre o envelhecimento (Torres, Camargo, Bousfield \& Silva, 2015; Camargo, Contarello, Wachelke, Morais \& Piccolo, 2014; Quéniart \& Charpentier, 2012; Moreira, Coutinho, Queiroga, Matos \& Silva, 2012; Mendes, Alves, Silva, Paredes \& Rodrigues, 2012; Torres, 2010; Wachelke \& Contarello, 2010), estudos envolvendo o rejuvenescimento ainda são incipientes, abordando de forma geral o pensamento social sobre o objeto (Castro, Aguiar et al, 2016; Torres, 2010; Teixeira, Settembre \& Leal, 2007) e buscando tecer as primeiras relações entre rejuvenescimento e suas práticas (Aguiar, 2016; Castro, Antunes et al, 2016; Berri et al, 2016). Desta forma, a presente pesquisa buscou verificar as representações sociais sobre o rejuvenescimento para mulheres de meia-idade e sua relação com práticas corporais relativas a este objeto, enfatizando atitudes, crenças e normas como elementos representacionais envolvidos na orientação e justificação destas práticas.

\section{Método}

Participantes: As participantes deste estudo foram 100 mulheres de meia-idade, com idade entre 38 e 62 anos, recrutadas entre trabalhadoras de instituições públicas. A média de idade das participantes foi de 49 anos e 8 meses ( $D P=6$ anos e 9 meses). Houve predominância de participantes com ensino superior completo $(71,7 \%)$, casadas ou em união estável (53,5\%) e com renda familiar superior a $\mathrm{R} \$ 7.000,00$ mensais $(47,5 \%)$.

Instrumento: Os dados foram coletados por meio de observação indireta, com a utilização de um questionário estruturado, auto aplicado em situação coletiva, contendo os seguintes blocos de questões:

1) Representações Sociais do Rejuvenescimento: As representações sociais do rejuvenescimento foram identificadas por meio de quatro questões:

a) Dimensão atitudinal: as atitudes em relação às práticas de rejuvenescimento foram identificadas por meio de 8 itens escalares do tipo Likert. Foram considerados 8 tipos de práticas corporais de rejuvenescimento, divididas em duas modalidades: práticas de rejuvenescimento não invasivas, compreendendo práticas alimentares e exercícios físicos voltados ao rejuvenescimento da aparência, uso de cosméticos/cremes anti-idade e uso de tinturas para cobrir cabelos brancos; e práticas minimamente invasivas e invasivas, incluindo aplicação de toxina botulínica (Botox) e de preenchimento cutâneo, realização de peeling químico e cirurgia plástica. Os itens apresentavam afirmações como "Agrada-me a possibilidade de praticar exercícios físicos para ter uma aparência mais jovem" e "Sou favorável à utilização de Botox para obter uma aparência mais jovem" e as participantes foram solicitadas a indicarem, em uma escala de 7 pontos (1- discordo totalmente a 7concordo totalmente), o nível de concordância com a afirmação. As práticas foram selecionadas de acordo com sua popularidade no Brasil (SBPC, 2009) e considerando dados de outros estudos em psicologia social, no qual foram associadas, pelas pessoas, ao rejuvenescimento (Aguiar, 2016; Castro, Antunes, et al, 2016; Torres, 2010).

b) Crenças sobre práticas de rejuvenescimento: as crenças foram mensuradas por meio de 6 itens escalares, tipo Likert (1 discordo totalmente a 5 concordo totalmente). Foram considerados 3 itens com sentido de aprovação em relação às práticas de rejuvenescimento e 3 com sentido de reprovação, tais como "Uma aparência envelhecida é 
sinal de descuido e desleixo consigo próprio" e "Eu considero fúteis as pessoas que procuram aparentar ser mais jovens do que são".

c) Dimensão normativa: As normas subjetivas foram apreendidas por meio de quatro itens escalares do tipo Likert, com 7 pontos, em que se buscou identificar a adesão normativa das participantes em relação a cinco contextos sociais: família, parceiro (a), amigos, pessoas próximas e pessoas ao redor. Ex: Se meus familiares me indicassem o uso de recursos para rejuvenescer, eu (1- nunca aceitaria a 7-sempre aceitaria); De um modo geral, o que as pessoas ao meu redor pensam que eu deva fazer para rejuvenescer: (1-Não tem muita importância a 7 -Tem muita importância). A norma pessoal foi mensurada por meio de um item escalar, tipo Likert: Para mim uma aparência rejuvenescida é algo: (1-Sem nenhuma importância a 7 - Extremamente importante).

d) Tarefa de escolha de palavras: Foi solicitado que as participantes assinalassem, em uma lista contendo 19 palavras, as 5 que mais consideravam relacionadas a palavra Rejuvenescimento, classificando as palavras escolhidas em ordem decrescente de importância.

2) Práticas de rejuvenescimento: Foram apresentadas duas questões. A primeira teve o objetivo de identificar as práticas corporais de rejuvenescimento já adotadas e para tal solicitou-se às participantes assinalarem em uma lista contendo diversas práticas corporais, as que já haviam sido adotadas pelo menos uma vez. A segunda questão visou apreender a intenção para adotar futuramente as práticas corporais de rejuvenescimento consideradas no estudo, sendo a intenção para cada prática mensurada por meio de um item escalar do tipo Likert (1-nunca faria a 5- com certeza faria).

3) Questões complementares: Consistiram em dados de caracterização das participantes (idade, escolaridade, estado civil e renda familiar).

Análise de dados: Os dados foram submetidos à análise estatística descritiva (distribuição de frequências absoluta e relativa, medidas de dispersão e tendência central) e relacional (teste de associação qui-quadrado, teste de diferenças de média -teste $\mathrm{t} e$ correlação de Person), por meio do software SPSS (Statistical Package Social Sciences), versão 17.0. Para a análise estatística relacional, foi realizada a verificação de normalidade dos dados recorrendo ao teste Kolmogorov-Smirnov. Constatada a não normalidade da distribuição, optou-se em realizar a análise por meio de estatística não paramétrica (Teste Kruskal Wallis).

Quanto às atitudes, além da análise dos itens de forma individual, também foi realizada uma análise considerando o agrupamento das práticas em duas modalidades: as consideradas não invasivas e as consideradas minimamente invasivas e invasivas. Os valores obtidos na análise dos itens agrupados permitiram a classificação das participantes em subgrupos, formados com base em suas atitudes em relação às duas modalidades de práticas. As participantes que obtiveram escore superior à mediana $(M d=4)$ nos itens agrupados das duas modalidades de práticas foram consideradas muito favoráveis às práticas corporais de rejuvenescimento. As que obtiveram escore superior à mediana no agrupamento das práticas não invasivas e escore igual ou inferior à mediana no agrupamento das práticas minimamente invasivas e invasivas foram consideradas parcialmente favoráveis às práticas. E as que obtiveram escore igual ou inferior à mediana nos itens agrupados das duas modalidades de práticas foram consideradas desfavoráveis às práticas corporais de rejuvenescimento. 
Aspectos éticos: Em conformidade com a norma 466/2012 do Conselho Nacional de Saúde, a pesquisa foi submetida à avaliação do Comitê de Ética em Pesquisas com Seres Humanos da Universidade Federal de Santa Catarina, obtendo aprovação sob o Parecer Consubstanciado no 370.109. As participantes foram esclarecidas quanto ao caráter anônimo e voluntário da participação e assinaram um Termo de Consentimento Livre e Esclarecido.

\section{Resultados}

Em relação às atitudes frente às práticas corporais de rejuvenescimento, a maior parte das mulheres apresentou atitudes positivas: 47 foram consideradas muito favoráveis, 32 parcialmente favoráveis e uma menor proporção de participantes $(n=21)$ foi classificada como desfavorável às práticas de rejuvenescimento. As atitudes em relação a cada prática podem ser visualizadas na Tabela 1.

Tabela 1 - Atitudes em relação às práticas de rejuvenescimento

\begin{tabular}{|c|c|c|c|c|c|c|}
\hline & \multicolumn{6}{|c|}{ Atitudes frente às práticas de rejuvenescimento } \\
\hline & \multicolumn{6}{|c|}{ Posicionamento frente às práticas } \\
\hline & \multicolumn{2}{|c|}{ Desfavoráveis } & \multicolumn{2}{|c|}{ P. Favoráveis } & \multicolumn{2}{|c|}{ M. Favoráveis } \\
\hline & $M$ & $D P$ & $M$ & $D P$ & $M$ & $D P$ \\
\hline \multicolumn{7}{|l|}{ Práticas não invasivas } \\
\hline Práticas alimentares & 4,00 & 2,24 & 5,84 & 1,85 & 6,06 & 1,39 \\
\hline Exercícios físicos & 3,95 & 2,38 & 4,72 & 1,92 & 6,00 & 1,32 \\
\hline Cosméticos/cremes anti-idade & 3,00 & 2,19 & 5,09 & 2,24 & 6,40 & 1,21 \\
\hline Tingimento cabelos brancos & 2,62 & 2,22 & 5,12 & 2,24 & 6,62 & 1,03 \\
\hline \multicolumn{7}{|c|}{ Práticas minimamente invasivas/invasivas } \\
\hline Botox & 1,29 & 0,72 & 1,28 & 1,42 & 5,70 & 1,61 \\
\hline Preenchimento cutâneo & 1,62 & 1,46 & 1,91 & 2,11 & 5,85 & 1,25 \\
\hline Peeling químico & 1,76 & 1,45 & 3,06 & 1,61 & 6,28 & 1,19 \\
\hline Cirurgia plástica & 1,67 & 1,24 & 2,50 & 1,61 & 5,51 & 1,61 \\
\hline
\end{tabular}

Nota: P. Favoráveis: parcialmente favoráveis / M. Favoráveis: muito favoráveis

Conforme a Tabela 01, "práticas alimentares" foi a única prática de rejuvenescimento na qual nenhum dos grupos de participantes apresentou atitude negativa. As práticas alimentares, o tingimento de cabelos brancos e o uso de cosméticos/cremes anti-idade foram práticas em que as participantes parcialmente favoráveis apresentaram atitudes mais positivas, enquanto que, entre as muito favoráveis, houve destaque para tingimento de cabelos brancos, cosméticos/cremes anti-idade e peeling químico. A diferença nas médias entre os grupos foi estatisticamente significativa em todas as práticas consideradas: práticas alimentares $\left[\chi^{2}(2)=16,51 ; p<0,001\right]$, exercícios físicos $\left[\chi^{2}(2)=17,53 ; p<0,001\right]$, cosméticos $\left[\chi^{2}(2)=35,88 ; p<0,001\right]$, tingimento de cabelos brancos $\left[\chi^{2}(2)=37,15 ; p<0,001\right]$, Botox $\left[\chi^{2}(2)=75,37 ; p<0,001\right]$, cirurgia plástica $\left[\chi^{2}(2)=55,04 ; p<0,001\right]$, peeling químico $\left[\chi^{2}(2)=59,99, p<0,001\right]$, preenchimento cutâneo $\left[\chi^{2}(2)=63,41 ; p<0,001\right]$. 
Tabela 2 - Crenças em relação às práticas de rejuvenescimento

\begin{tabular}{|c|c|c|c|c|c|c|}
\hline \multirow[t]{3}{*}{ Item } & \multicolumn{6}{|c|}{ Posicionamento } \\
\hline & \multicolumn{2}{|c|}{ Desfavoráveis } & \multicolumn{2}{|c|}{ P. Favoráveis } & \multicolumn{2}{|c|}{ M. Favoráveis } \\
\hline & $M$ & $D P$ & $M$ & $D P$ & $M$ & $D P$ \\
\hline $\begin{array}{l}\text { Uma aparência envelhecida é sinal de descuido e desleixo } \\
\text { consigo próprio. }\end{array}$ & 2,48 & 1,12 & 2,68 & 1,27 & 3,37 & 1,30 \\
\hline $\begin{array}{l}\text { Eu acho que as pessoas que buscam o rejuvenescimento só } \\
\text { estão preocupadas com a aparência. }\end{array}$ & 2,86 & 1,28 & 2,42 & 1,08 & 2,05 & 1,20 \\
\hline $\begin{array}{l}\text { Toda pessoa que tem amor próprio deve buscar } \\
\text { estratégias para ficar mais jovem. }\end{array}$ & 2,34 & 1,51 & 2,53 & 1,32 & 3,83 & 1,07 \\
\hline $\begin{array}{l}\text { Eu considero fúteis as pessoas que procuram aparentar ser } \\
\text { mais jovens do que são. }\end{array}$ & 3,26 & 1,39 & 2,95 & 1,21 & 1,82 & 1,10 \\
\hline $\begin{array}{l}\text { As pessoas que buscam o rejuvenescimento procuram } \\
\text { melhorar sua qualidade de vida. }\end{array}$ & 3,09 & 1,34 & 3,84 & 1,08 & 4,49 & 0,69 \\
\hline $\begin{array}{l}\text { As pessoas que buscam o rejuvenescimento não aceitam o } \\
\text { próprio envelhecimento. }\end{array}$ & 4,09 & 0,89 & 3,00 & 1,19 & 2,40 & 1,23 \\
\hline Total da medida $(\alpha=0,72)$ & 2,60 & 0,69 & 3,11 & 0,65 & 3,90 & 0,63 \\
\hline
\end{tabular}

Quanto às crenças referentes às práticas de rejuvenescimento, considerando o total da medida, verifica-se uma crença positiva a respeito destas práticas entre as participantes muito favoráveis às práticas, uma crença próxima à neutralidade entre as parcialmente favoráveis e uma crença negativa entre as participantes desfavoráveis $\left[\chi^{2}(2)=39,75 ; p<\right.$ $0,001]$.

Considerando de forma específica os itens, a crença de que a aparência envelhecida denota descuido e desleixo é compartilhada pelas participantes muito favoráveis, enquanto que as participantes desfavoráveis e parcialmente favoráveis tendem a discordar da mesma. As diferenças foram estatisticamente significativas $\left[\chi^{2}(2)=8,87 ; p<0,05\right]$. Observa-se consenso entre todas as participantes em discordar sobre a busca pelo rejuvenescimento ser suscitada por uma preocupação exclusiva com a aparência, no entanto as mulheres muito favoráveis demonstraram discordar mais desta crença em comparação com as parcialmente favoráveis e desfavoráveis $\left[\chi^{2}(2)=7,56 ; p<0,05\right]$. Também se observou consenso geral em relação ao rejuvenescimento ser considerado uma estratégia para a melhoria da qualidade de vida, no entanto com maior concordância por parte das participantes muito favoráveis em comparação às parcialmente favoráveis e desfavoráveis $\left[\chi^{2}(2)=22,19 ; p<0,001\right]$. A crença sobre a busca pelo rejuvenescimento consistir em uma expressão de amor próprio é compartilhada pelas mulheres muito favoráveis às práticas, enquanto que as parcialmente favoráveis e desfavoráveis tendem a discordar da mesma. As diferenças foram estatisticamente significativas $\left[\chi^{2}(2)=23,66 ; p<0,001\right]$. A crença que atrela 0 rejuvenescimento à futilidade é compartilhada somente pelas participantes desfavoráveis, já que as muito favoráveis tendem a discordar da mesma e as parcialmente favoráveis a se manterem próximas à neutralidade $\left[\chi^{2}(2)=23,29 ; p<0,001\right]$. As mulheres desfavoráveis também tendem a acreditar que a busca pelo rejuvenescimento é suscitada pela não aceitação do envelhecimento, enquanto que as participantes muito favoráveis tendem a discordar desta crença e as parcialmente favoráveis a se manterem neutras sobre a mesma $\left[\chi^{2}(2)=25,18 ; p<0,001\right]$.

Quanto à dimensão normativa das representações sociais, observaram-se diferenças entre os subgrupos de participantes quanto à adesão às normas subjetivas (Tabela 3). Tomando como referência o total da medida, as participantes muito favoráveis 
demonstraram alta adesão às normas subjetivas, enquanto que as parcialmente favoráveis e desfavoráveis baixa adesão $\left[\chi^{2}(2)=43,88 ; p<0,001\right]$. Os resultados em relação a cada contexto social podem ser visualizados na Tabela 3.

Tabela 3 - Normas subjetivas por posicionamento frente às práticas de rejuvenescimento.

\begin{tabular}{lcccccc}
\hline Item & \multicolumn{4}{c}{ Posicionamento } \\
\cline { 2 - 8 } & \multicolumn{2}{c}{ Desfavoráveis } & \multicolumn{2}{c}{ P. Favoráveis } & \multicolumn{2}{c}{ M. Favoráveis } \\
\hline Expectativas de familiares & $M$ & $D P$ & $M$ & $D P$ & $M$ & $D P$ \\
Expectativas do(a) parceiro (a) & 2,90 & 1,95 & 4,37 & 1,50 & 5,96 & 1,39 \\
Expectativas de amigos & 3,19 & 1,57 & 4,34 & 1,51 & 5,94 & 1,43 \\
Expectativas de pessoas próximas & 2,43 & 1,57 & 3,59 & 1,85 & 4,72 & 1,66 \\
Expectativas de pessoas ao redor & 1,95 & 1,20 & 3,15 & 1,67 & 4,87 & 1,75 \\
\hline Total da medida $(\boldsymbol{\alpha}=\mathbf{0 , 8 1})$ & 2,04 & 1,63 & 3,00 & 1,80 & 3,89 & 2,04 \\
\hline
\end{tabular}

Considerando individualmente os itens, os familiares e o parceiro exercem maior influência normativa sobre as mulheres, principalmente entre as muito favoráveis, no entanto somente estas últimas demonstraram concordar com as expectativas de amigos e pessoas próximas. As participantes desfavoráveis, por sua vez, tendem a discordar muito das expectativas das pessoas de todos os contextos sociais considerados. As diferenças foram estatisticamente significativas [familiares: $\chi^{2}(2)=36,45 ; p<0,001$; parceiro: $\chi^{2}(2)=36,12 ; p<$ 0,001; amigos: $\chi^{2}(2)=22,53 ; p<0,001$; pessoas próximas: $\chi^{2}(2)=36,06 ; p<0,001$ ].

Nota-se que, quando se considerava grupos sem uma discriminação específica, tal como "pessoas ao seu redor", a relevância do contexto normativo perde a importância também para as participantes muito favoráveis, embora as mesmas tenham apresentado médias superiores às desfavoráveis e as parcialmente favoráveis e a diferença ter sido estatisticamente significativa $\left[\chi^{2}(2)=12,53 ; p<0,05\right]$.

Em relação à norma pessoal, verifica-se que as mulheres desfavoráveis consideram a aparência rejuvenescida pouco importante $(M=3,05 ; D P=1,93)$, enquanto que as parcialmente favoráveis se mostram neutras neste aspecto $(M=4,03 ; D P=1,49)$ e as muito favoráveis consideram-na muito importante, apresentando alta adesão à norma $(M=5,61$; $D P=1,25)$. A diferença foi estatisticamente significativa $\left[\chi^{2}(2)=31,58 ; p<0,001\right]$.

Quanto aos resultados da tarefa de escolha de palavras, a Tabela 4 apresenta as palavras apresentadas às participantes, organizadas de acordo com a frequência em que foram associadas ao rejuvenescimento. 
Tabela 4 - Distribuição de frequência das palavras escolhidas referentes ao termo "rejuvenescimento" por posicionamento.

\begin{tabular}{|c|c|c|c|c|c|c|c|c|}
\hline & \multirow{3}{*}{ Palavras } & \multirow{3}{*}{$\begin{array}{c}\text { Total } \\
(\mathrm{N}=100)\end{array}$} & \multicolumn{6}{|c|}{ Posicionamento } \\
\hline & & & \multicolumn{2}{|c|}{$\begin{array}{l}\text { Desfavoráveis } \\
\quad(n=21)\end{array}$} & \multicolumn{2}{|c|}{$\begin{array}{l}\text { P. Favoráveis } \\
(n=32)\end{array}$} & \multicolumn{2}{|c|}{$\begin{array}{l}\text { M. Favoráveis } \\
(n=47)\end{array}$} \\
\hline & & & Freq. & OI & Freq. & OI & Freq. & $\mathrm{OI}$ \\
\hline \multirow{13}{*}{ 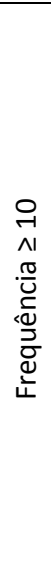 } & Autoestima & 73 & 16 & 2,75 & 20 & 2,30 & 37 & 2,11 \\
\hline & Saúde & 56 & 11 & 2,00 & 14 & 1,43 & 31 & 1,84 \\
\hline & Bem-estar & 50 & 11 & 3,09 & 16 & 3,00 & 23 & 2,78 \\
\hline & Exercícios físicos & 49 & 09 & 3,22 & 12 & 4,50 & 28 & 3,39 \\
\hline & Estado de espírito & 37 & 11 & 2,36 & 13 & 2,84 & 13 & 2,84 \\
\hline & Vitalidade & 32 & 10 & 3,70 & 13 & 2,54 & 09 & 3,55 \\
\hline & Beleza & 29 & 02 & 5,00 & 12 & 3,75 & 15 & 4,13 \\
\hline & Autocuidado & 29 & 07 & 3,43 & 07 & 3,14 & 15 & 2,67 \\
\hline & Felicidade & 28 & 07 & 2,14 & 09 & 3,44 & 12 & 2,75 \\
\hline & Vaidade & 23 & 03 & 4,33 & 08 & 3,25 & 12 & 4,08 \\
\hline & Estética & 23 & 01 & 5,00 & 08 & 3,12 & 14 & 4,21 \\
\hline & Cirurgia plástica & 15 & 01 & 3,00 & 06 & 3,83 & 08 & 4,00 \\
\hline & Cobrança social & 15 & 04 & 3,00 & 06 & 1,67 & 05 & 4,80 \\
\hline \multirow{6}{*}{ 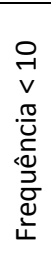 } & Artificial & 06 & 02 & 4,00 & 03 & 3,67 & 01 & 2,00 \\
\hline & Ilusão & 05 & 02 & 3,50 & 03 & 2,67 & - & - \\
\hline & Mídia & 05 & 02 & 2,00 & 03 & 4,67 & - & - \\
\hline & Exagero & 04 & 01 & 4,00 & 02 & 3,50 & 01 & 1,00 \\
\hline & Risco & 03 & - & - & 03 & 3,67 & - & - \\
\hline & Futilidade & 01 & 01 & 5,00 & - & - & - & - \\
\hline
\end{tabular}

Nota: (Freq.) Frequência; (OI) Ordem de importância.

Considerando todas as participantes da amostra, nota-se uma representação social do rejuvenescimento ligada a dimensões físicas e subjetivas. Em termos de proporção, a autoestima (Desfavoráveis=76,19\%; Parcialmente Favoráveis=62,5\%; Muito Favoráveis $=78,7 \%$ ), a saúde (Desfavoráveis $=52,4 \%$; Parcialmente Favoráveis $=43,8 \%$; Muito Favoráveis $=66,0 \%$ ), os exercícios físicos (Desfavoráveis $=42,9 \%$; Parcialmente Favoráveis=37,5\%; Muito Favoráveis=59,6\%), o bem-estar (Desfavoráveis=52,4\%; Parcialmente Favoráveis $=50,0 \%$; Muito Favoráveis $=48,9 \%$ ) e o estado de espírito (Desfavoráveis=52,4\%; Parcialmente Favoráveis=40,6\%; Muito Favoráveis=27,7\%), foram as palavras mais escolhidas pelas participantes, seguidos de vitalidade (Desfavoráveis=47,6\%; Parcialmente Favoráveis $=40,6 \%$ e Muito Favoráveis $=19,1 \%$ ), felicidade (Desfavoráveis=33,3\%; Parcialmente Favoráveis=28,1\%; Muito Favoráveis $=25,5 \%$ ) e autocuidado (Desfavoráveis=33,3\%; Parcialmente Favoráveis=21,9\%; Muito Favoráveis=31,9\%), o que sugere que o rejuvenescimento é associado predominantemente à preservação da funcionalidade do corpo e a questões referentes ao bem-estar psíquico. A ausência de associação significativa entre quase todas as palavras e o posicionamento frente às práticas de rejuvenescimento mostra que se trata de uma representação compartilhada tanto pelas participantes favoráveis quando desfavoráveis a essas práticas. A vitalidade foi o único elemento associado a um grupo específico, uma vez que, embora também tenha sido mencionada pelas participantes parcialmente favoráveis e muito favoráveis, foi mais associada ao rejuvenescimento pelas participantes desfavoráveis $\left[\chi^{2}(2)=7,01 ; p<0,05\right]$.

Os aspectos estéticos foram pouco atrelados ao rejuvenescimento pelas participantes desfavoráveis, visto um pequeno número destas terem escolhido as palavras "beleza" 
(9,5\%), "vaidade" (14,3\%), "estética" (4,8\%) e "cirurgia plástica" $(4,8 \%)$, elementos mais considerados pelas participantes parcialmente favoráveis e muito favoráveis: beleza $(37,5 \%$ contra $31,9 \%)$, vaidade ( $25 \%$ contra $25,5 \%$ ), estética ( $25 \%$ contra $29,8 \%$ ), cirurgia plástica (18,8\% contra 17\%). Contudo, a autoestima foi mencionada pelos três grupos, aludindo a aspectos pragmáticos da representação que ligam o rejuvenescimento às práticas corporais promotoras de beleza física, que, por sua vez, contribuem para o bem-estar subjetivo. Por fim, aspectos normativos associados à manutenção da beleza, como a cobrança social, se mostraram mais salientes para as participantes desfavoráveis e parcialmente favoráveis (Desfavoráveis=19\%; Parcialmente Favoráveis=18,8\% e Muito Favoráveis=10,6\%) quando as mesmas pensavam sobre o rejuvenescimento, embora a diferença não tenha sido estatisticamente significativa ao se considerar os três posicionamentos.

Quanto à ordem de importância, a saúde figura como o elemento mais importante para todas as participantes. A autoestima se mostra o segundo elemento mais importante para as participantes parcialmente favoráveis e muito favoráveis, enquanto que as desfavoráveis valorizam mais aspectos subjetivos como a felicidade e o estado de espírito. Por outro lado, os elementos considerados menos importantes são os que envolvem aspectos estéticos, como a vaidade, a estética, a beleza e a cirurgia plástica, renegados a um posto secundário ao serem comparados com aspectos mais funcionais e subjetivos, o que ocorre principalmente entre as participantes muito favoráveis.

Tabela 5 - Adoção e intenção de adotar práticas de rejuvenescimento

\begin{tabular}{|c|c|c|c|c|c|c|}
\hline & \multicolumn{6}{|c|}{ Práticas de rejuvenescimento adotadas pelo menos uma vez } \\
\hline & Total & Desfavoráveis & \multicolumn{2}{|c|}{ P. Favoráveis } & \multicolumn{2}{|c|}{ M. Favoráveis } \\
\hline & Freq. & Freq. & \multicolumn{2}{|c|}{ Freq. } & \multicolumn{2}{|c|}{ Freq. } \\
\hline \multicolumn{7}{|l|}{ Práticas não invasivas } \\
\hline Tingimento cabelos brancos & 71 & 09 & \multicolumn{2}{|c|}{23} & \multicolumn{2}{|c|}{39} \\
\hline Cosméticos/cremes & 69 & 08 & \multicolumn{2}{|c|}{17} & \multicolumn{2}{|c|}{44} \\
\hline Exercícios físicos & 68 & 09 & \multicolumn{2}{|c|}{18} & \multicolumn{2}{|c|}{41} \\
\hline Práticas alimentares & 03 & - & \multicolumn{2}{|c|}{01} & \multicolumn{2}{|c|}{02} \\
\hline \multicolumn{7}{|l|}{ Práticas minim. invasivas/invasivas } \\
\hline Cirurgia plástica & 22 & - & \multicolumn{2}{|c|}{03} & \multicolumn{2}{|c|}{19} \\
\hline Botox & 19 & - & \multicolumn{2}{|c|}{-} & \multicolumn{2}{|c|}{19} \\
\hline Peeling químico & 20 & 01 & & & 17 & \\
\hline Preenchimento cutâneo & 11 & 01 & & & 10 & \\
\hline Outros & & & & & & \\
\hline Outros procedimentos estéticos & 14 & 03 & & & $0 \varepsilon$ & \\
\hline Nunca utilizou quaisquer práticas & 08 & 08 & & & - & \\
\hline & Inte & ção em adotar & prátic & e rejuve & iescimen & \\
\hline & & Posicionam & ento $\mathrm{fr}$ & às práti & cas & \\
\hline & Desfavor & veis & P. Fav & eis & M. Favo & veis \\
\hline & $M$ & $D P$ & $M$ & $D P$ & $M$ & $D P$ \\
\hline Práticas não invasivas & & & & & & \\
\hline Práticas alimentares & 3,42 & 1,40 & 3,87 & 1,07 & 4,34 & 0,84 \\
\hline Exercícios físicos & 3,81 & 1,29 & 4,23 & 0,83 & 4,88 & 0,38 \\
\hline Cosméticos/cremes anti-idade & 2,71 & 1,45 & 3,97 & 1,23 & 4,85 & 0,41 \\
\hline Tingimento cabelos brancos & 2,58 & 1,51 & 4,29 & 1,22 & 4,92 & 0,45 \\
\hline Práticas minim. invasivas/invasiv & & & & & & \\
\hline Botox & 1,14 & 0,36 & 1,59 & 0,76 & 4,03 & 1,22 \\
\hline Preenchimento cutâneo & 1,19 & 0,51 & 1,62 & 0,87 & 3,91 & 1,17 \\
\hline Peeling químico & 1,57 & 0,75 & 2,34 & 1,38 & 3,98 & 1,13 \\
\hline Cirurgia plástica & 1,43 & 0,68 & 1,92 & 0,81 & 3,48 & 1,16 \\
\hline
\end{tabular}


Considerando as práticas corporais de rejuvenescimento já adotadas (Tabela 5), quase totalidade das participantes (92\%) declarou já ter utilizado práticas não invasivas pelo menos uma vez, enquanto somente $38 \%$ declararam o uso de práticas minimamente invasivas e invasivas. Todas as pessoas que declararam o uso de práticas de rejuvenescimento minimamente invasivas e invasivas também referiram o uso de práticas não invasivas.

Ao se considerar o posicionamento, a maior parte das participantes muito favoráveis às práticas $(n=32)$ referiu já ter utilizado práticas minimamente invasivas e invasivas, enquanto que a minoria entre as parcialmente favoráveis $(n=4)$ e desfavoráveis $(n=2)$ referiu tal adesão. Todas as participantes muito favoráveis e parcialmente favoráveis às práticas de rejuvenescimento referiram adesão às práticas não invasivas. Não foi encontrada diferença estatisticamente significativa em relação ao estado civil, mas foi encontrada em relação à renda familiar, no qual as participantes com renda superior a $\mathrm{R} \$ 7.000,00$ declararam maior adoção a algumas práticas minimamente invasivas como o Botox $\left[\chi^{2}(1)=5,40 ; p<0,05\right]$ e peeling químico $\left[\chi^{2}(1)=10,63 ; p<0,001\right]$ quando comparadas às participantes com renda familiar igual ou abaixo deste valor.

Quando questionadas sobre a intenção em adotar práticas de rejuvenescimento, observa-se a manutenção do mesmo padrão relativo à adoção passada, com maior intencionalidade de adoção de práticas não invasivas (Tabela 5). Quanto às práticas minimamente invasivas e invasivas, observa-se que somente as mulheres muito favoráveis às práticas de rejuvenescimento se mostraram dispostas a adotá-las, cujas médias apresentadas indicam uma possibilidade situada entre "talvez utilizaria" e "provavelmente utilizaria", com exceção da prática de Botox em que as médias indicam uma intencionalidade direcionada à "provavelmente utilizaria". A diferença de média entre os três grupos referente a práticas desta modalidade foi estatisticamente significativa: cirurgia plástica $\left[\chi^{2}(2)=47,25 ; p<0,001\right]$, Botox $\left[\chi^{2}(2)=65,03 ; p<0,001\right]$, preenchimento cutâneo $\left[\chi^{2}(2)=62,96\right.$; $p<0,001]$ e peeling químico $\left[\chi^{2}(2)=42,10 ; p<0,001\right]$.

As práticas não invasivas, por sua vez, destacam-se por apresentarem alta possibilidade de adoção também entre as participantes parcialmente favoráveis, indicadas como recursos rejuvenescedores que elas "provavelmente" ou "com certeza" utilizariam. As práticas alimentares e exercícios físicos foram as únicas as quais as participantes desfavoráveis às práticas de rejuvenescimento considerariam utilizar. A maior intencionalidade em realizar as quatro práticas não invasivas consideradas foi encontrada entre as participantes muito favoráveis. A diferença foi estatisticamente significativa: práticas alimentares $\left[\chi^{2}(2)=8,14 ; p<0,05\right]$, exercícios físicos $\left[\chi^{2}(2)=27,62 ; p<0,001\right]$, cosméticos/cremes anti-idade $\left[\chi^{2}(2)=39,79 ; p<0,001\right]$ e tingimento de cabelos brancos $\left[\chi^{2}(2)=47,56 ; p<0,001\right]$.

Não foram encontradas diferenças estatisticamente significativas entre a intenção para adotar práticas e estado civil, mas o foi em relação à variável renda familiar, em que as participantes com renda familiar superior a $\mathrm{R} \$ \mathbf{7 . 0 0 0 , 0 0}$ declararam maior intenção para adotar a cirurgia plástica [t(95)=2,40; $p<0,05]$, Botox [t(96)=2,59; $p<0,05]$, preenchimento cutâneo [t(94)=2,07, $p<0,05]$, peeling químico [t(95)=3,52; $p<0,01]$ e tingimento de cabelos brancos $[\mathrm{t}(86,35)=2,55 ; \quad p<0,05]$ do que as participantes com renda igual ou inferior a este valor.

No que se refere à relação entre as representações sociais e práticas, realizou-se o cruzamento das questões referentes aos elementos representacionais de atitudes, crenças e 
normas sobre práticas de rejuvenescimento com o tipo de prática adotada e a intenção em adotar essas práticas.

Quanto à relação entre atitudes e práticas, verificou-se uma atitude mais positiva sobre as práticas minimamente invasivas e invasivas entre as participantes que referiram já terem adotado essa modalidade de práticas quando comparadas às participantes que declararam apenas o uso de práticas não invasivas: cirurgia plástica $[\mathrm{t}(90)=4,49 ; p \leq 0,001]$, Botox [t $(90)=5,16 ; p \leq 0,001]$, preenchimento cutâneo [t(90)=6,16; $p \leq 0,001]$, peeling químico $\mathrm{t}(90)=5,66 ; p \leq 0,001]$. Aquelas também apresentaram atitudes mais positivas do que estas últimas em relação à prática de tinturas de cabelo $t(81,9)=4,49 ; p \leq 0,05]$. Ao se considerar a intenção em adotar práticas de rejuvenescimento, foram realizadas correlações entre os itens que mensuravam as atitudes referentes a cada prática de rejuvenescimento e os que mensuravam a intenção para adotá-las futuramente. Identificaram-se correlações positivas entre as variáveis, o que indica que uma maior intenção em se engajar em determinada prática está relacionada a uma atitude mais positiva em relação a esta mesma prática. A magnitude da correlação foi forte com as práticas: cirurgia plástica $[r=0,82 ; p<$ 0,001], Botox [r=0,88; $p<0,001]$, preenchimento cutâneo $[r=0,79 ; p<0,001]$, peeling químico [ $r=0,84 ; p<0,001]$ e tingimento de cabelos brancos $[r=0,83 ; p<0,001]$; e moderada com as práticas: exercícios físicos $[r=0,51 ; p<0,001]$, cosméticos/cremes anti-idade $[r=0,60$; $p<0,001]$ e práticas alimentares $[\mathrm{r}=0,40 ; p<0,001]$.

Quanto à relação entre crenças e práticas, tanto as participantes que referiram já terem adotado práticas minimamente invasivas e invasivas quanto as que adotaram apenas práticas não invasivas compartilham uma crença positiva em relação às práticas de rejuvenescimento, no entanto as primeiras apresentam uma crença mais positiva $(M=3,73$; $D P=0,80)$ do que as segundas $(M=3,28 ; D P=0,74)$ e esta diferença foi estatisticamente significativa $[\mathrm{t}(90)=2,72 ; p<0,001]$. Já quando se considera a intenção para adotar práticas de rejuvenescimento, verificou-se que uma maior intencionalidade está associada a crenças mais positivas, o que foi observado em relação a todas as práticas de rejuvenescimento consideradas no estudo: cirurgia plástica $[r=0,63, p<0,01]$, Botox $[r=0,68, p<0,01]$, preenchimento cutâneo $[r=0,54 ; \quad p<0,01]$, peeling químico $[r=0,69 ; p<0,01]$, cosméticos/cremes anti-idade $[\mathrm{r}=0,47 ; p<0,01]$, tingimento de cabelos brancos $[\mathrm{r}=0,44 ; p<$ $0,01]$, exercícios físicos $[r=0,34 ; p<0,01]$, práticas alimentares $[r=0,25 ; p<0,05]$.

No que se refere à relação entre normas e práticas, nota-se que as participantes que já adotaram práticas minimamente invasivas e invasivas demonstram uma maior adesão às normas subjetivas $(M=4,76 ; D P=1,35)$ quando comparadas às participantes que só utilizaram práticas não invasivas $(M=3,83 ; D P=1,33)$. A diferença foi estatisticamente significativa $[\mathrm{t}(90)=3,27 ; p<0,01]$. Já quanto à intenção de engajamento nessas práticas, observou-se que uma maior intencionalidade se mostrou associada a uma maior adesão às normas subjetivas, o que foi identificado por meio da correlação positiva entre os itens da primeira variável com o total da medida da segunda: cirurgia plástica $[r=0,60 ; p<0,01]$, Botox $[r=0,58$; $p<0,01]$, preenchimento cutâneo [ $\mathrm{r}=0,57 ; p<0,01]$, peeling químico [ $\mathrm{r}=0,55 ; p<0,01]$, cosméticos/cremes anti-idade $[r=0,54 ; p<0,01]$, tingimento de cabelos brancos $[r=0,47 ; p<$ $0,01]$, exercícios físicos $[r=0,27 ; p<0,01]$, práticas alimentares $[r=0,50 ; p<0,01]$.

No que tange à relação entre práticas e norma pessoal, as mulheres que referiram já ter adotado práticas minimamente invasivas e invasivas demonstraram maior adesão a esta norma $(M=5,46 ; D P=1,51)$ do que as mulheres que referiram utilizar apenas práticas não invasivas $(M=4,23 ; D P=1,62)$. A diferença foi estatisticamente significativa [ $\mathrm{t}(92)=3,72 ; p<$ 
0,001]. Além disso, também se identificou uma correlação entre norma pessoal e intenção para adotar as práticas de rejuvenescimento consideradas no estudo, indicando que uma maior intencionalidade para se engajar nestas práticas está relacionada a uma maior valorização da jovialidade da aparência. A força da correlação foi fraca para as práticas alimentares [ $r=0,30 ; p<0,01]$, exercícios físicos [ $r=0,36, p<0,01]$ e tinturas para cabelos $[r=0,38, p<0,01]$ e moderada para cirurgia plástica $[r=0,49, p<0,01]$, preenchimento cutâneo [ $r=0,49 ; p<0,01]$, Botox $[r=0,53 ; p<0,01]$, peeling químico $[r=0,53 ; p<0,01]$ e cosméticos/cremes anti-idade $[\mathrm{r}=0,58 ; p<0,01]$.

\section{Discussão}

Este estudo teve como objetivo analisar a relação entre representações sociais do rejuvenescimento com práticas corporais relativas a este objeto. Por serem consideradas variáveis envolvidas na orientação dos comportamentos, utilizou-se o conceito de atitude, crenças e normas como pontos de interface entre os dois construtos.

Considerando as atitudes, este estudo mostrou que a maior parte das participantes foi considerada favorável a práticas corporais de rejuvenescimento, embora com distintos níveis de intensidade. Ainda que a beleza e a juventude consistam em valores amplamente compartilhados, esse resultado evidencia que as atitudes em relação às práticas de rejuvenescimento podem se organizar em torno de sistemas simbólicos variados, que por sua vez vão ilustrar diferentes sentidos que as mulheres atribuem ao processo de envelhecer. Considerando que é por meio das crenças que as pessoas encontram argumentos para justificar e defender suas atitudes (Lima, 2006; Fishbein \& Ajzen, 1975; Ajzen, 1991), diferentes posicionamentos sobre essas práticas indicam a existência de distintas crenças sobre as mesmas, implicando em atributos valorizados de forma positiva ou negativa (Stroebe \& Stroebe, 1995). As atitudes muito positivas parecem se sustentar em crenças referentes às práticas corporais de rejuvenescimento que as associam à melhoria da qualidade de vida e como uma forma de autocuidado e amor próprio, bem como a repulsão pela visibilidade do envelhecimento físico, considerando-o sinal de descuido e desleixo. Por outro lado, as atitudes negativas parecem estar alicerçadas em crenças que atrelam o rejuvenescimento corporal à futilidade e a uma forma de negação do envelhecimento. No entanto, a existência de um posicionamento intermediário entre ser favorável e ser desfavorável às práticas sugerem que a adesão a essas crenças pode se dar em níveis variados de intensidade e ter relação com o tipo de prática adotada.

Como também encontrado em outros estudos (Castro, Antunes et al, 2016, Teixeira et al., 2007; Clarke \& Griffin, 2007), observou-se uma maior aceitação das práticas corporais de rejuvenescimento não invasivas em relação às minimamente invasivas e invasivas, o que se refletiu tanto no aspecto afetivo quanto comportamental. As atitudes em relação às primeiras foram mais positivas do que a das segundas, assim como a intencionalidade de adesão às práticas e a sua adoção concreta. Por outro lado, o engajamento em práticas minimamente invasivas e invasivas se deu em menor proporção, como também encontrado por Ehlinger-Martin et al. (2015), predominando entre as mulheres muito favoráveis às práticas, que também foram as únicas a considerar utilizá-las no futuro. A maior aceitação de práticas não invasivas pode estar relacionada à maior sensação de segurança que elas transmitem, tanto em relação à saúde quanto à aparência (Castro, Antunes et al, 2016; Ehlinger-Martin et al., 2015; Teixeira et al., 2007; Clarke et al, 2007), bem como serem 
consideradas respostas menos drásticas contra o envelhecimento (Muise \& Desmarais, 2010). Assim, ao avaliarem as práticas de rejuvenescimento, as mulheres parecem ponderar as perdas e ganhos das mesmas, buscando a beleza sem prejuízo da saúde.

Considerando a relação entre crenças, atitudes e comportamento (Ajzen \& Fishbein, 1975), observou-se que crenças e atitudes mais positivas em relação às práticas de rejuvenescimento se associaram tanto a maior adoção quanto a maior intencionalidade para adotar essas práticas. O conjunto de avaliações carregadas de afeto são particularmente importantes em transformar atitude em ação (Fraser, 1994), e essa avaliação é regulada por uma dimensão social, derivando dos valores e normas do grupo de referência (ParalesQuenza \& Vizicaíno-Gutiérrez, 2007). Portanto, apesar das atitudes em relação a um objeto serem consideradas um intermediário importante na relação entre representações sociais do grupo e a emissão de comportamento ou práticas (Camargo \& Bousfield, 2014; Wachelke \& Camargo, 2007), elas se articulam em um campo social regulado por sistemas normativos, em que normas, crenças, valores pessoais modulam a representação social do objeto (Doise, 1985; 2001).

A adesão a elementos normativos das representações sociais do rejuvenescimento se mostrou uma condição que favorece o engajamento em práticas corporais referentes a este objeto. Observou-se que uma maior adesão às pressões sociais pela jovialidade da aparência e maior internalização dessas pressões, demostrada pela valorização da jovialidade física, se mostrou relacionado à maior favorabilidade às práticas, maior intenção de futuramente adotá-las e engajamento prévio em práticas mais intervencionistas. A família e o parceiro revelaram-se os contextos normativos mais significativos para as participantes, principalmente para as muito favoráveis às práticas, que se mostraram muito motivadas a corresponder às expectativas destas pessoas em relação ao rejuvenescimento. Outros estudos também identificaram uma saliência da pressão social de familiares (Camargo et al., 2010) e do parceiro (Szymanski \& Cash, 1995) em relação à importância da aparência e satisfação corporal. Assim, esses resultados demonstram que a dependênca afetiva influencia a relação que as mulheres estabelecem com seus corpos, cuja necessidade de aceitação por parte do outro parece se constituir em um elemento importante na construção da autoimagem (Jodelet, 1994), predispondo-as a adoção de práticas corporais de rejuvenescimento.

Os diferentes posicionamentos referentes às práticas corporais de rejuvenescimento e suas relações com a valorização da jovialidade da aparência, revelam como as mulheres negociam suas identidades femininas dentro das ideias culturais de beleza. Embora o corpo e a aparência representem uma parte importante da identidade de gênero das mulheres, as pessoas do mesmo grupo ou categoria social podem não endossar as mesmas normas ou podem internalizá-las em níveis variados (Moscovici, 2011). Isso permite que a importância atribuída ao corpo possa ser relativizada entre as mulheres dentro da uma sociedade, ou seja, corresponder a um aspecto normativo internalizado em níveis variados. Considerando que as normas mais internalizadas são consideradas as que mais explicam o comportamento (Bertoldo, 2013; Th Øgersen, 2006), distintos níveis de internalização normativa referente à jovialidade física podem estar envolvidos na adoção a diferentes práticas de rejuvenescimento.

Este estudo também mostrou que o rejuvenescimento é representado não apenas como um objeto de beleza, mas também de saúde e ligado a condições psicológicas, representação compartilhada de forma consensual por todas as mulheres do estudo, 
confirmando os achados de outros estudos (Berri et al, 2016; Torres, 2010). A saúde é apontada como o aspecto mais importante em relação ao enfrentamento do envelhecimento, o que sugere a primazia de aspectos funcionais ao se pensar em rejuvenescimento. Embora com ênfase sobre seus efeitos sobre a aparência, as práticas alimentares e os exercícios físicos foram práticas aceitáveis tanto para mulheres favoráveis quanto desfavoráveis às práticas de rejuvenescimento, o que pode ser justificado pelos benefícios que também possam proporcionar à saúde. $O$ engajamento nestas práticas parece se sustentar em crenças subjacentes que atrelam o envelhecimento à doença e a decrepitude física, condição que pode ser atenuada ou acentuada em função das escolhas realizadas ao longo da vida. Desta forma, o conhecimento sobre o rejuvenescimento parece se revelar amparado em sistemas de valores comuns e divergentes, sendo a combinação particular das diversas cognições sustentadas por esses sistemas, em função das inserções sociais das participantes (favoráveis ou desfavoráveis às práticas), que orientam a adesão a certas práticas em detrimentos de outras.

É pertinente mencionar ainda que embora os elementos valorativos das representações sociais do rejuvenescimento se mostrem como contingências que predispõe a adoção de práticas corporais de rejuvenescimento, não necessariamente eles garantem a manifestação concreta destas ações. Neste estudo, a correspondência entre atitudecomportamento foi maior em relação às práticas não invasivas do que as minimamente invasivas e invasivas, já que entre essas últimas nem todas as mulheres com atitudes positivas declararam as terem adotado. Rouquette (1998) defende que as representações sociais contribuem para explicar as práticas, mas a ocorrência das mesmas também depende de outros fatores, como por exemplo, circunstâncias ambientais ou fatores individuais. Por envolverem um investimento financeiro maior, a adoção de certas práticas pode estar sujeita às condições socioeconômicas das mulheres, o que foi evidenciado neste estudo. As mulheres com maior renda familiar fizeram maior uso de algumas práticas como o Botox e peeling químico, bem como demonstraram maior intencionalidade para adotar práticas minimamente invasivas e invasivas. As práticas não invasivas, por outro lado, ao serem menos dispendiosas em termos financeiros, seriam mais acessíveis à população com menor poder aquisitivo, justificando, em parte, a maior adesão a estas práticas.

Por fim, conclui-se que os marcos valorativos e normativos das representações sociais do rejuvenescimento constituem-se em sistemas de significação centrais que estão envolvidos na adoção de práticas corporais de rejuvenescimento, consequentemente se constituindo em elementos representacionais que sustentam a adoção destas práticas. As atitudes se estabelecerem como núcleos do sistema representacional e organizam as cognições associadas ao rejuvenescimento, adquirindo sua força valorativa em função da adesão a sistemas normativos ligadas a um padrão de beleza, por sua vez associado à juventude. As normas subjetivas e pessoal foram evidenciadas como âncoras das representações sociais do rejuvenescimento para as mulheres com posicionamento favorável às práticas, uma vez que elas parecem reconhecer a importância da beleza e jovialidade da aparência para as relações interpessoais que estabelecem.

Quanto às limitações do estudo é importante mencionar que a abordagem de um amplo conjunto de práticas corporais de rejuvenescimento no mesmo estudo impossibilitou uma exploração mais aprofundada das relações entre representações sociais e práticas. Os resultados sugerem que práticas não invasivas e minimamente invasivas e invasivas são simbolizadas de formas distintas entre as mulheres, com maior aceitação das primeiras em 
detrimento das demais. Tal fato demonstra que a relação entre as práticas corporais de rejuvenescimento com as representações sociais que as sustentam é muito diversificada, o que dificulta a proposição de um modelo único. Ademais, o universo de práticas de rejuvenescimento adotado nesta pesquisa não esgota todas as intervenções de rejuvenescimento disponíveis, de forma que os posicionamentos favoráveis e desfavoráveis devem ser considerados parciais.

Este estudo evidenciou que a relação entre pensamento leigo e ação é caracterizada pela complexidade, portanto, o entendimento desta relação vai exigir outros estudos que aprofundem o papel dos aspectos valorativos e normativos das representações na orientação de práticas de rejuvenescimento, bem como considerem nesta relação a influência de outras variáveis de ordem individual e societária.

\section{Referências}

Abric, J-C.(1994). Introduction. In J-C. Abric (Org.). Pratiques sociales et représentations (pp. 07-09). Paris: Presses Universitaires de France.

Aguiar, A. (2016). Envelhecimento e rejuvenescimento em contextos intergrupais antagônicos: representações sociais e práticas corporais. Tese de Doutorado, Universidade Federal de Santa Catarina, Florianópolis, Brasil.

Almeida, A. M. O.; Santos, M. F. S., \& Trindade, Z. A. (2000). Representações e práticas sociais: contribuições teóricas e dificuldades metodológicas. Temas em Psicologia da SPB, 8(3), 257-267.

Ajzen, I. (1991). The Theory of Planned Behavior. Organizational Behavior and Human Decision Processes, 50, 179-211.

Ajzen, I. (2001). Nature and operation of attitudes. Annual Review Psychology, 52, 27-58.

Ajzen, I. (2005). Attitudes, personality and behavior. New York: Open University Press.

Ajzen, I. (2006). Constructing a TpB questionnaire: Conceptual and methodological considerations. Recuperado de: http://www.people.mass.edu/aizen/tpb.html.

Ajzen, I., \& Madden, T. J. (1986). Prediction of goal-directed behavior: attitudes, intentions and perceived behavioral control. Journal of Experimental Social Psychology, 22, 453-474.

Ajzen, I., \& Fishbein, M. (1975). Understanding attitudes and predicting social behavior. New Jersey: PrenticeHall.

Berri, B., Castro, A., \& Camargo, B. V. (2016). Representações sociais relacionadas às práticas de rejuvenescimento. Psicologia em Pesquisa-UFJF, 10(2), 21-30.

Bertoldo, R. B. (2013). A valorização social do pró-ambientalismo enquadrado por normas formais: uma análise psicossocial comparativa entre Brasil e Portugal. Tese de Doutorado, Instituto Universitário de Lisboa, Lisboa, Portugal.

Camargo, B. V., \& Bousfield, A. S. B. (2014). Em direção a um modelo explicativo da relação entre representações sociais e práticas relativas à saúde: a ideia de adesão representacional. In E. M. Q. O. Chamon; P. A. Guareschi \& P. H. F. Campos. Textos e debates em representação social (pp. 261-284). Porto Alegre: ABRAPSO.

Camargo, B. V., Contarello, A., Wachelke, J. F. R., Morais, D. X., \& Piccolo, C. (2014). Representações Sociais do Envelhecimento entre Diferentes Gerações no Brasil e na Itália. Psicologia em Pesquisa (UFJF), 8(2), 179 188.

Camargo, B. V., Justo, A. M., \& Jodelet, D. (2010). Normas, representações sociais e práticas corporais. Interamerican Journal of Psychology, 44(3), 449-457. 
Campos, P. H. F. (2003). O estudo das relações entre práticas sociais e representações. Estudos Goiânia, 30(1), 51-59.

Castro, A., Aguiar, A., Berri, B., \& Camargo, B. V. (2016). Representações sociais do rejuvenescimento na mídia impressa. Temas em Psicologia, 24(1), 117-130.

Castro, A., Antunes, L., Brito, A. M. M., \& Camargo, B. V. (2016). Representações sociais do envelhecimento e do rejuvenescimento para mulheres que adotam práticas de rejuvenescimento. Psico, 47(4), 319-330.

Chasteen, A. L., Bashir, N. Y., Gallucci, C., \& Visekruna, A. (2011). Age and antiaging technique influence reactions to age concealment. The Journals of Gerontology, Series B: Psychological Sciences and Social Sciences, 66(6), 719-724.

Clarke, L. H., \& Griffin, M. (2008). Visible and invisible ageing: beauty work as a response to ageism. Ageing \& Society, 28, 653-674.

Clarke, L. H., \& Griffin, M. (2007). The body natural and the body unnatural: Beauty work and aging. Journal of Aging Studies, 21, 187-201.

Clarke, L. H., Repta, R., \& Griffin, M. (2007). Non-surgical cosmetic procedures: Older women's perceptions and experiences. Journal of Women and Aging, 19(3/4), 69-87.

Doise, W. (1985). Les représentations sociales: définition d'un concept. Connexions, 45, 243-253.

Doise, W. (2001) Atitudes e representações sociais. In D. Jodelet (Org.). As representações sociais (pp. 187-203). Rio de Janeiro: EDUERJ.

Doise, W. (2014). Sistema e Metassistema. In: A. M. O. Almeida, M. F. Souza \& Z. A. Trindade (Eds.). Teoria das representações sociais -50 anos (pp. 123-156). Rio de Janeiro: Techno Politik Editora.

Ehlinger-Martin, A., Cohen-Letessier, A., Taïeb, M., Azoulay, E., \& du Crest, D. (2015). Women's attitudes to beauty, aging, and the place of cosmetic procedures: insights from the QUEST Observatory. Journal of Cosmetic Dermatology, 1-6, 89-94.

Fishbein, M., \& Ajzen, I. (1975). Attitudes towards objects as predictors of single and multiple behavioral criteria. Psychological Review, 81, 59-74.

Flament, C. (2001). Estrutura e dinâmica das representações sociais. In D. Jodelet (Org.). As representações sociais (pp. 173-186). Rio de Janeiro: UERJ.

Fraser, C. (1994). Attitudes, social representations and widespread beliefs. Papers on Social Representations, 3(1), 1-138.

Guimelli, C. (1998). Chasse et nature en languedoc: étude de la dynamique d'une représentation sociale. Paris: L'Harmattan.

Jodelet, D. (1994). Le corps, la persone et autrui. In S. Moscovici (Org.). Psychologie sociale des relations à autrui (pp. 41-68). Paris: Nathan.

Jodelet, D. (1989). Les representationes sociales. Paris: Press Universitaires de France.

Jodelet, D. (2001). Representações sociais: um domínio em expansão. In D. Jodelet (Org.). As representações sociais (pp. 17-41). Rio de Janeiro: Ed. UDUERJ

Lheureux, F., LoMonaco, G., \& Guimelli, C. (2011). Entre representations sociales et intentions de pratiques: l'implication. Interamerican Journal of Psychology, 45(1), 61-76.

Lima, L. P. (2006). Atitudes: estrutura e mudança. In J. Vala \& M. B. Monteiro (Orgs). Psicologia Social (pp. $187-$ 225). Lisboa: Fundação Calouste Gulbenkian.

Mamontoff, A. M. (2008). Dynamique de la fonction identitaire dês représentations sociales dans le cas d'une rencontre entre deux cultures. Anuario de Psicología, 39(2), 249-268.

Mendes, C. K. T. T., Alves, M. S. C. F., Silva, A. O., Paredes, M. A. S., \& Rodrigues, T. P. (2012) Representações sociais de trabalhadores da atenção básica de saúde sobre envelhecimento. Revista Gaúcha de Enfermagem, 33(3), 148-155.

Moliner, P., \& Tafani, E. (1997). Attitudes and social representations: a theoretical and experimental approach. European Journal of Social Psychology, 27, 687-702. 
Moreira, M. A. S. P.; Coutinho, N. J. M.; Queiroga, A. Q. S. G.; Matos, M. Y. C., \& Silva, A. O. (2012). Social Representations about aging. The FIEP Bulletin, Special edition, 82, 363-365.

Morot, R., \& Sales-Wuillemin, E. (2008). Effet des pratiques et des connaissancessur la représentation sociale d'un objet: application à l'hygiène hospitalière. Revue Internationale de Psychologie Sociale, 21(4), 89114.

Moscovici, S. (1961/1978). A representação social da psicanálise. Rio de Janeiro: Zahar Editores.

Moscovici, S. (2011). Psicologia das minorias ativas. Petrópolis: Ed. Vozes.

Muise, A. \& Desmarais, S. (2010). Women's Perceptions and Use of “Anti-Aging” Products. Sex Roles, 63, 126137.

Muth, J. L., \& Cash, T. F. (1997). Body-image attitudes: What difference does gender make? Journal of Applied Social Psychology, 27,1438-1452.

Neiva, E. R., \& Mauro, T. G. (2011). Atitudes e mudança de atitude. In E. R. Neiva \& C.V. Torres. Psicologia Social no Brasil: Principais temas e vertentes (pp.163-195). Porto Alegre: Artmed.

Parales-Quenza, C. J. \& Vizcaíno-Gutiérrez, M. (2007). Las relaciones entre actitudes y representaciones sociales: elementos para uma integración conceptual. Revista Latinoamericana de Psicología, 39(2), 351361.

Pereira, C. P., \& Penalva, G. (2011). “Mulher-madonna” e outras mulheres: um estudo antropológico sobre a juventude aos 50 anos. In M. Goldenberg (Org). Corpo, envelhecimento e felicidade (pp. 133-158). Rio de Janeiro: Civilização Brasileira.

Quéniart, A., \& Charpentier, M. (2012). Older women and their representations of old age: a qualitative analysis. Ageing and Society, 32(6),983 1007.

Rouquette, M-L. (1998). Representações e práticas sociais: alguns elementos teóricos. In A. S. P Moreira \& D. C. de Oliveira (Orgs.). Estudos interdisciplinares de representação social (pp. 39-46). Goiânia: AB Editora.

Sá, C. P. (1994). Sur les relations entre représentations sociales, pratiques socio-culturelles et comportement. Papers on Social Representations, 3, 40-46.

Slevec, J., \& Tiggemann, M. (2010). Attitudes toward cosmetic surgery in middle-aged women: body image, aging axiety and the media. Psychology of Women Quarterly, 34(1), 65-74.

SBCP -Sociedade Brasileira de Cirurgia Plástica (2009). Cirurgia Plástica no Brasil. Pesquisa Datafolha, Janeiro. Recuperado de:_http://www2.cirurgiaplastica.org.br/wpcontent/uploads/2012/11/pesquisa2009.pdf. Acessado em 20 de fevereiro de 2013.

Stroebe, W., \& Stroebe, M. S. (1995). Psicologia Social e Saúde. Lisboa: Instituto Piaget.

Stuart-Hamilton I. (2002). A Psicologia do envelhecimento: uma introdução (3ạ ed.). Porto Alegre: Artmed.

Szymansky, M. L., \& Cash, T. F. (1995). Body-image disturnances and self-discrepancy theory: expansion of the body-image ideals questionnaire. Journal of Social and Clinical Psychology, 14(2), 134-146.

Teixeira, M. C. T. V., Franchin, A. B. B., Durso, F. A., Donati, L. B., Facin, M. M., \& Pedreschi, P. T. (2007). Envelhecimento e rejuvenescimento: um estudo de representação social. Revista Brasileira de Geriatria e Gerontologia, 10(1), 49-71.

Teixeira, M. C. T. V., Settembre, F. M. \& Leal, S. B. (2007). A survey of women's social representations of aging and rejuvenation. The Spanish Journal of Psychology, 10(1), 104-114.

Thøgersen, J. (2006). Norms for environmentally responsible behaviour: An extended taxonomy. Journal of Environmental Psychology, 26, 247-61.

Torres, T.L. (2010). Pensamento social sobre envelhecimento, idoso e rejuvenescimento para diferentes grupos etários. Tese de Doutorado. Universidade Federal de Santa Catarina, Florianópolis, SC.

Torres, T. L., Camargo, B. V., Bousfield, A. B., \& Silva, A. O. (2015) Representações sociais e crenças normativas sobre envelhecimento. Ciência e Saúde Coletiva, 20(12), 3621-3630.

Vala, J. (2006). Representações sociais e psicologia social do conhecimento cotidiano. In J. Vala \& B. Monteiro (Orgs). Psicologia Social (7ạ ed) (pp. 457-502). Lisboa: Fundação Calouste Gulbenkian. 
Wachelke, J. F. R., \& Camargo, B. V. (2007). Representações sociais, representações individuais e comportamento. Revista Interamericana de Psicologia, 41(3): 379-390.

Wachelke, J. F. R., \& Contarello, A. (2010). Social representations on aging: Structural differences concerning age group and cultural context. Revista Latinoamericana de Psicologia,42(3), 367-380.

Wagner, W. (2015). Representation in action. In G. Sammut; E. Andreouli; G. Gaskell, \& J. Valsiner (Eds). The Cambridge Handbook of Social Representations (pp. 12-28). Cambridge, UK: Cambridge University Press.

Wagner, W. (2003). People in Action and Social Representation: A Comment on Jaan Valsiner's "Theory of Enablement". Papers on Social Representations, 12, 8.1-8.7.

Yaworsky, A., Daniels, S., Tully, S., Beddingfield, F., Kowalski, J., Fitzgerald, K., Somogyi, C., \& Burgess, S.M. (2014). The impact of upper facial lines and psychological impact of crow's feet lines: content validation of the Facial Line Outcomes (FLO-11) Questionnaire. Journal of Cosmetic Dermatology, 13, 297-306.

Apresentação: 15/03/2017 Aprovação: 15/06/2017 